TITLE:

\title{
Slow dynamics near glass transitions in thin polymer films
}

AUTHOR(S):

Fukao, K; Miyamoto, Y

CITATION:

Fukao, K ...[et al]. Slow dynamics near glass transitions in thin polymer films. Physical Review E 2001, 64(1): 011803.

ISSUE DATE:

2001-07

URL:

http://hdl.handle.net/2433/50238

RIGHT:

Copyright 2001 American Physical Society 
PHYSICAL REVIEW E, VOLUME 64, 011803

\title{
Slow dynamics near glass transitions in thin polymer films
}

\author{
Koji Fukao* and Yoshihisa Miyamoto \\ Faculty of Integrated Human Studies, Kyoto University, Kyoto 606-8501, Japan \\ (Received 25 December 2000; published 19 June 2001)
}

\begin{abstract}
The $\alpha$ process (segmental motion) of thin polystyrene films supported on glass substrate has been investigated in a wider frequency range from $10^{-3} \mathrm{~Hz}$ to $10^{4} \mathrm{~Hz}$ using dielectric relaxation spectroscopy and thermal expansion spectroscopy. The relaxation rate of the $\alpha$ process increases with decreasing film thickness at a given temperature above the glass transition. This increase in the relaxation rate with decreasing film thickness is much more enhanced near the glass transition temperature. The glass transition temperature determined as the temperature at which the relaxation time of the $\alpha$ process becomes a macroscopic time scale shows a distinct molecular weight dependence. It is also found that the Vogel temperature has a thickness dependence, i.e., the Vogel temperature decreases with decreasing film thickness. The expansion coefficient of the free volume $\alpha_{f}$ is extracted from the temperature dependence of the relaxation time within the free volume theory. The fragility index $m$ is also evaluated as a function of thickness. Both $\alpha_{f}$ and $m$ are found to decrease with decreasing film thickness.
\end{abstract}

DOI: 10.1103/PhysRevE.64.011803

PACS number(s): 64.70.Pf, 68.60.-p, 77.22.Gm

\section{INTRODUCTION}

Glass transitions and related dynamics in thin polymer films have widely been investigated by various experimental techniques $[1,2]$. One of the major motivations of such studies is to investigate the finite size effects of glass transitions that could be related to the cooperative motion in supercooled liquids near the glass transitions. For this purpose, the glass transition temperature $T_{\mathrm{g}}$ in thin films has been measured by using several experimental methods such as ellipsometry $[3,4]$, neutron reflectivity measurement [5], positron annihilation lifetime spectroscopy (PALS) [6], dielectric measurement $[7,8]$. Although contradicting experimental results on $T_{\mathrm{g}}$ have been reported in the literatures, some common features seem to appear among recent experimental results [2]. In many cases, the glass transition temperatures in thin films are lower than those of the bulk system if there is no strong attractive interaction between polymers and substrate $[3,4]$. In particular, the glass transition temperature in freely standing films is much lower than that in thin polymer films supported on substrate $[9,10]$.

The dynamics of the $\alpha$ process (segmental motion) of polymers in thin films have also been investigated by some techniques. Second harmonic generation reveals that the distribution of relaxation times broadens with decreasing film thickness, while the average relaxation time of the $\alpha$ process remains constant for supported films of a random copolymer [11]. In the case of freely standing films of polystyrene, photon correlation spectroscopy studies indicate that the relaxation behavior of the $\alpha$ process in thin films is similar to that of bulk samples of polystyrene, except for the reduction of the $\alpha$ relaxation time [12]. Because there are only a few experimental observations on the dynamics of thin polymer films, the dynamical properties of the $\alpha$ process have not yet been clarified in thin polymer films.

\footnotetext{
*Author to whom correspondence should be addressed. Electronic address: fukao@phys.h.kyoto-u.ac.jp
}

Measurements of chain mobility in thin polymer films supported on substrate have also been done by using fluorescence recovery after photobleaching [13] and dynamic secondary ions mass spectroscopy [14]. A decrease in the chain mobility of the polymer melt is observed in thin films or near the substrate by such experiments. This result appears to be inconsistent with the reduction in $T_{\mathrm{g}}$ and also in the relaxation rate of segmental motions.

In the previous papers $[7,8]$, we reported that $T_{\mathrm{g}}$ for thin polystyrene films supported on glass substrate can be determined as the crossover temperature at which the temperature dependence of the electric capacitance changes drastically and that the dynamics of the $\alpha$ process can be determined from the frequency dependence of the complex dielectric constant of the films. We confirmed that $T_{\mathrm{g}}$ decreases with decreasing film thickness in the same way as observed by ellipsometry [3] and that the temperature $T_{\alpha}$, at which the dielectric loss shows the peak at a given frequency of applied electric field (from $10^{2} \mathrm{~Hz}$ to $10^{4} \mathrm{~Hz}$ ), is also lower in thin films than that in the bulk system, and the thickness dependence of $T_{\alpha}$ clearly depends on the molecular weight of the polymer. Because the glass transition temperature is directly connected with the dynamics of the $\alpha$ process, the temperatures $T_{\alpha}$ and $T_{\mathrm{g}}$ are expected to have similarity in the properties including the molecular weight dependence. However, no experimental results that support the existence of the molecular weight dependence of $T_{\mathrm{g}}$ in thin supported polymer films have so far been reported [2], in contrast with the case of freely standing thin films, where the glass transition temperature is found to strongly depend on the molecular weight of polymers $[9,10,15]$.

In this paper, we investigate the dynamics of $\alpha$ process in thin films of polystyrene, especially the thickness dependence of the dynamics for a wider frequency range, including lower frequencies corresponding to the glass transition region, in order to clarify the dependence of the relaxation time of the $\alpha$ process, $\tau_{\alpha}$, and $T_{\mathrm{g}}$ on thickness and molecular weight. For this purpose, we adopt a new technique, thermal expansion spectroscopy (TES) [16-18], in addition to dielec- 
tric relaxation spectroscopy (DES). For polar materials with strong dielectric relaxation strength, DES is a powerful method that can cover very wide frequency range from $10^{-4} \mathrm{~Hz}$ to $10^{12} \mathrm{~Hz}$ by itself [19-21]. Because polystyrene is less polar material, however, it is difficult to obtain significant dielectric loss signals in lower frequency ranges by DES. In such lower frequency range, TES is a powerful tool for nonpolar materials.

On the basis of the observed relaxation rates as functions of temperature for the thin films with various thicknesses, we obtain the Vogel temperature and thermal expansion coefficient of the free volume. The fragility index is also obtained as a function of thickness and the non-Arrhenius behavior of the $\alpha$ process in thin polymer films is discussed in comparison with that in small molecules confined in nanopores.

\section{EXPERIMENTAL DETAILS}

\section{A. Sample preparation}

Atactic polystyrene (a-PS) used in this study was purchased from Scientific Polymer Products, Inc. $\left(M_{\mathrm{w}}=2.8\right.$ $\left.\times 10^{5}\right)$, the Aldrich Co., Ltd. $\left(M_{\mathrm{w}}=1.8 \times 10^{6}, M_{\mathrm{w}} / M_{\mathrm{n}}\right.$ $=1.03)$, and Polymer Source, Inc. $\left(M_{\mathrm{w}}=6.67\right.$ $\left.\times 10^{6}, M_{\mathrm{w}} / M_{\mathrm{n}}=1.22\right)$, where $M_{\mathrm{w}}$ and $M_{\mathrm{n}}$ are the weight average and the number average of the molecular weights, respectively. Thin films of a-PS with various thicknesses were prepared on an Al-deposited glass substrate using a spin-coat method from a toluene solution of a-PS. The thickness was controlled by changing the concentration of the solution. After annealing at $343 \mathrm{~K}$ in the vacuum system for several days to remove solvents, Al was vacuum deposited once more to serve as an upper electrode. Thickness of each film is evaluated from the electric capacitance at room temperature using the equation of the flat-plate condenser on the assumption that dielectric permittivity $\epsilon^{\prime}$ does not depend on thickness, i.e., $\epsilon^{\prime}=2.8$ [22]. This assumption may not appear to be a good assumption for very thin films. However, in our previous papers $[7,8]$, we showed that the glass transition temperature $T_{\mathrm{g}}$ determined as the temperature at which the temperature coefficient of the electric capacitance changes discontinuously agrees very well with that observed by ellipsometry even for very thin films, as mentioned in the Introduction. In our measurements, thickness is determined from the electric capacitance on the basis of the above assumption. These results may lead to the validity of the assumption that $\epsilon^{\prime}$ is independent of thickness for the thickness range investigated here.

Thin films prepared according to the above procedure are located in air, in a hand-made sample cell. The temperature is measured by an Chromel-Alumel thermocouple attached on the back side of glass substrate. Two successive thermal cycles prior to measurements are performed on prepared thin films before the data acquisition starts in order to relax asspun films and obtain reproducible results. In the first thermal cycle the films are heated from room temperature to 343 $\mathrm{K}$ and then cooled down to room temperature. In the second thermal cycle the procedure is the same as in the first one except that the upper-limited temperature is $378 \mathrm{~K}$ (not $343 \mathrm{~K})$.
Even after measurements up to $378 \mathrm{~K}$, the samples were found not to suffer from dewetting. However, an optical microscope measurement showed that the surface of the upper electrode of the thin films was slightly rough after the measurements, while that of the lower electrode between the polymers and glass substrate remained smooth. This implies that the upper electrode does not affect the thermal properties of thin polymer films.

\section{B. Dielectric relaxation spectroscopy}

For dielectric relaxation measurements we use an LCR meter (HP4284A) in the range of frequency of applied electric field, $f_{E}$, from $20 \mathrm{~Hz}$ to $1 \mathrm{MHz}$. The measurements were performed during heating (cooling) processes between room temperature and $408 \mathrm{~K}$ at a heating (cooling) rate of 0.5 $\mathrm{K} / \mathrm{min}$. From the dielectric loss data in the wide temperature and frequency ranges we extracted the temperature $T_{\alpha}$ at which dielectric loss exhibits a peak at a given frequency. In order to avoid the effect of the extra loss peak due to the existence of a finite resistance within the upper electrode $[8,23]$, we utilized the dielectric data up to $10 \mathrm{kHz}$.

\section{Thermal expansion spectroscopy}

Thermal expansion spectroscopy is a new technique that has very recently been introduced in studies on slow dynamics in supercooled liquids by Bauer et al. [16]. In this method, a sinusoidal temperature modulation,

$$
T(t)=\langle T\rangle+T_{\omega} e^{i \omega t}
$$

is applied to the sample, and then the corresponding change in capacitance with the same angular frequency $\omega$ as the temperature modulation has,

$$
C^{\prime}(t)=\left\langle C^{\prime}\right\rangle-C_{\omega}^{\prime} e^{i(\omega t+\delta)},
$$

is detected within a linear response region. Here, $\omega=2 \pi f_{\mathrm{T}}$ and $f_{\mathrm{T}}$ is the frequency of temperature modulation, $\langle T\rangle$ is the average temperature, $\left\langle C^{\prime}\right\rangle$ is the averaged capacitance, $T_{\omega}$ is the amplitude of the temperature modulation with angular frequency $\omega, C_{\omega}^{\prime}$ is the amplitude of the response capacitance with the angular frequency $\omega$, and $\delta$ is the phase lag between the temperature modulation and the corresponding capacitance change. In the case of a flat-plate condenser, $C^{\prime}=\epsilon^{\prime} \epsilon_{0} S / d$, where $\epsilon^{\prime}$ is the permittivity of a-PS, $\epsilon_{0}$ is the permittivity of the vacuum, $S$ is the area of the electrode ( $S=8.0 \mathrm{~mm}^{2}$ in the present measurements), and $d$ is the thickness of the films. If there is no dynamical process involved, $\epsilon^{\prime} \approx \epsilon_{\infty}$, where $\epsilon_{\infty}$ is the permittivity in the highfrequency limit, and hence the capacitance change with temperature is directly connected with volume change in thin films via $\epsilon_{\infty}, S$, and $d$ as shown in the previous paper [8].

In the case of thin films of a-PS in which the area of the film surface remains constant with temperature change, the linear thermal expansion coefficient, $\alpha_{\mathrm{n}}$, normal to the film surface satisfies the following relation: 


$$
\alpha_{\mathrm{n}}=-\zeta \frac{1}{C^{\prime}\left(T_{s}\right)} \frac{d C^{\prime}}{d T}=\zeta \frac{1}{C^{\prime}\left(T_{s}\right)} \frac{C_{\omega}^{\prime}}{T_{\omega}} e^{i \delta},
$$

where $C^{\prime}(T)$ is the capacitance at temperature $T$ and $T_{s}$ is a standard temperature, $\zeta \approx 0.5$ for a-polystyrene [8].

As far as the response obeys the change in external field without any delay, the phase lag $\delta$ is zero. However, if there is any dynamical process with a characteristic time of the order of $1 / \omega$ in the system, $\delta$ is no longer zero, and hence, $\alpha_{\mathrm{n}}$ becomes a complex number. Hereafter, we denote $\alpha_{\mathrm{n}}$ by $\alpha_{\mathrm{n}}^{*}$, where

$$
\alpha_{\mathrm{n}}^{*}=\alpha_{\mathrm{n}}^{\prime}-i \alpha_{\mathrm{n}}^{\prime \prime}
$$

Here, $\alpha_{\mathrm{n}}^{\prime}$ and $\alpha_{\mathrm{n}}^{\prime \prime}$ are the real and imaginary parts of the complex thermal expansion coefficient.

In the present measurements the average temperature $\langle T\rangle$ is controlled to increase with a constant rate, $0.1 \mathrm{~K} / \mathrm{min}$ or $0.5 \mathrm{~K} / \mathrm{min}$. The amplitude $T_{\omega}$ is set between $0.2 \mathrm{~K}$ and $0.6 \mathrm{~K}$. For capacitance measurements in TES the frequency of the applied electric field was chosen to be $100 \mathrm{kHz}$ to avoid the interference with dielectric relaxations [16].

In both DES and TES measurements, we measure (complex) capacitance of thin films, and hence we use the same sample for the two different measurements. If $f_{T}=0$ and $f_{E}$ is varied at a fixed temperature, we obtain the data of the frequency dispersion of the dielectric constant. If $f_{E}$ is fixed to be a high frequency, for example, $100 \mathrm{kHz}$ and $f_{T}$ is varied at a given temperature, we are able to perform TES measurement on the same sample that is used for DES measurements. For this reason, we can obtain dynamical properties in a wider frequency range from $10^{-3} \mathrm{~Hz}$ to $10^{4} \mathrm{~Hz}$ even for less polar material such as a-PS by combining two powerful methods DES and TES.

\section{RESULTS AND DISCUSSION}

\section{A. Dynamics of the $\alpha$ process near the glass transition temperature}

Figure 1 displays the temperature dependence of both the real and imaginary parts $\alpha_{\mathrm{n}}^{\prime}$ and $\alpha_{\mathrm{n}}^{\prime \prime}$ measured by TES for thin films of a-PS with film thickness $18 \mathrm{~nm}$ and $247 \mathrm{~nm}$ and $M_{\mathrm{w}}=6.7 \times 10^{6}$. The modulation frequencies of temperature, $f_{T}$, are $2.1 \mathrm{mHz}$ and $16.7 \mathrm{mHz}$. The peak temperature $T_{\alpha}$ at which the imaginary part of $\alpha_{\mathrm{n}}^{*}$ has a maximum at a given frequency $f_{T}$ shifts to a lower temperature with decreasing film thickness. At the same time, the width in the temperature domain of the transition region between the glassy state and the liquid state becomes broader as the thickness decreases; $\sim 15 \mathrm{~K}$ for $d=247 \mathrm{~nm}$ and $\sim 30 \mathrm{~K}$ for $d=18 \mathrm{~nm}$. This corresponds to the fact that the distribution of the relaxation times of the $\alpha$ process becomes broader with decreasing film thickness. Our recent measurements of dielectric relaxation in thin films of a-PS have also revealed the broadening of the dielectric loss peak not only in the temperature domain but also in the frequency domain at frequencies above $100 \mathrm{~Hz}$ [8]. Ellipsometric studies also support the results observed in the dielectric measurements [24]. The
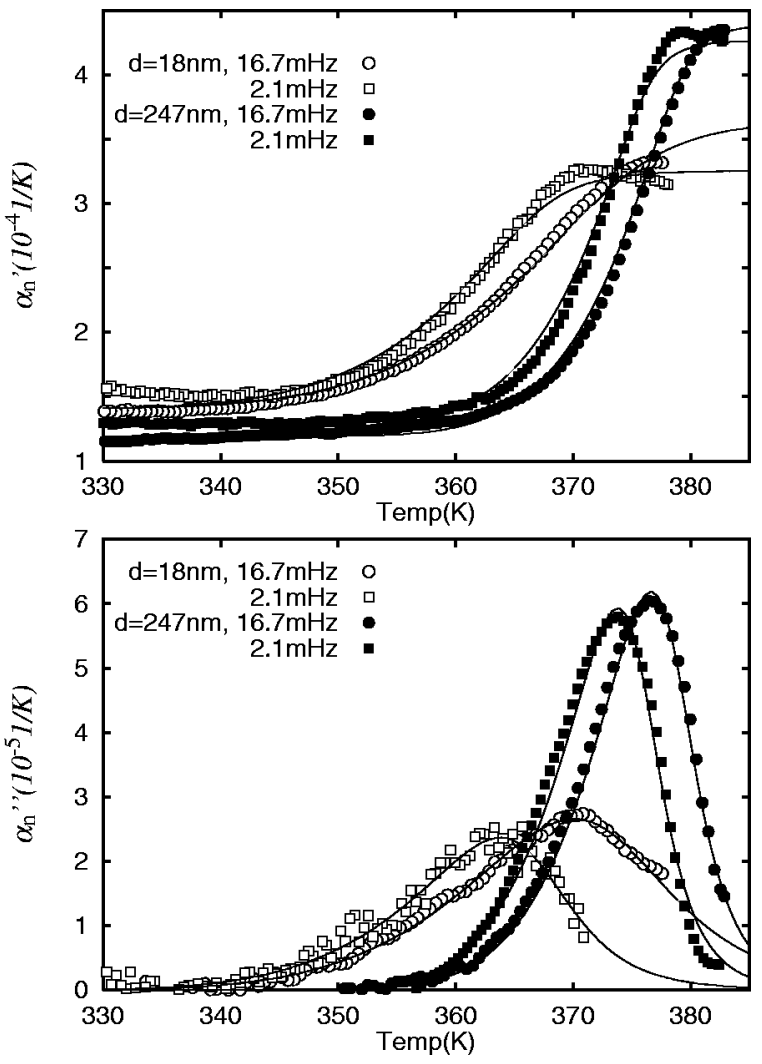

FIG. 1. Temperature dependence of complex linear thermal expansion coefficient $\alpha_{\mathrm{n}}^{*}$ for a-PS with film thickness $18 \mathrm{~nm}$ and 247 $\mathrm{nm}\left(f_{\mathrm{T}}=16.7 \mathrm{mHz}, 2.1 \mathrm{mHz}, M_{\mathrm{w}}=6.7 \times 10^{6}\right)$. The upper figure shows the real part of $\alpha_{\mathrm{n}}^{*}$ and the lower one, the imaginary part. Solid lines are calculated by using the HN equation and the VFT equation with the parameters listed in Table I.

broadening observed in the present TES measurements near $T_{\mathrm{g}}$ suggests that there is a distribution in $T_{\mathrm{g}}$ within the thin polymer layers and that the distribution becomes broader in thinner films.

The solid curves in Fig. 1 are reproduced by combining the following two equations. One is the Havriliak-Negami (HN) equation that can empirically describe the frequency dependence of susceptibility $\alpha^{*}(\omega)$ [25]:

$$
\alpha^{*}(\omega)=\frac{\Delta \alpha}{\left[1+\left(i \omega \tau_{\mathrm{HN}}\right)^{1-\alpha_{\mathrm{HN}}}\right]^{\beta_{\mathrm{HN}}}},
$$

where $\omega=2 \pi f, f$ is the frequency of the applied external field, $\Delta \alpha$ is the relaxation strength, $\tau_{\mathrm{HN}}$ is the relaxation time, $\alpha_{\mathrm{HN}}$ and $\beta_{\mathrm{HN}}$ are the shape parameters. The second one is the Vogel-Fulcher-Tammann (VFT) law that can widely be used to describe the temperature dependence of the relaxation time $\tau_{\alpha}$ of the $\alpha$ process:

$$
\tau_{\alpha}=\tau_{0} \exp \left(\frac{U}{T-T_{0}}\right)
$$

where $U$ is the apparent activation energy, $T_{0}$ is the Vogel temperature, and $\tau_{0}$ is the relaxation time at high temperatures [26]. In this analysis, it is assumed that the parameters 
TABLE I. Parameters for the HN equation and the VFT equation to obtain the solid curves in Fig. 1. The parameters are obtained for thin films of a-PS with $M_{\mathrm{w}}=6.7 \times 10^{6}$.

\begin{tabular}{lcccccc}
\hline \hline$d(\mathrm{~nm})$ & $f_{T}(\mathrm{~Hz})$ & $\alpha_{\mathrm{HN}}$ & $\beta_{\mathrm{HN}}$ & $\beta_{K}$ & $U(\mathrm{~K})$ & $T_{0}(\mathrm{~K})$ \\
\hline 18 & $1.67 \times 10^{-2}$ & $0.40 \pm 0.05$ & $0.22 \pm 0.03$ & 0.19 & 1887 & 312.7 \\
18 & $2.1 \times 10^{-3}$ & $0.38 \pm 0.07$ & $0.23 \pm 0.04$ & 0.20 & 1887 & 312.7 \\
247 & $1.67 \times 10^{-2}$ & $0.20 \pm 0.05$ & $0.29 \pm 0.03$ & 0.30 & 1733 & 324.0 \\
247 & $2.1 \times 10^{-3}$ & $0.23 \pm 0.06$ & $0.27 \pm 0.04$ & 0.27 & 1733 & 324.0 \\
\hline \hline
\end{tabular}

$\Delta \alpha, \alpha_{\mathrm{HN}}$, and $\beta_{\mathrm{HN}}$ in Eq. (5) are independent of temperature and that $\tau_{\mathrm{HN}}=\tau_{\alpha}$. The parameters used in Fig. 1 are listed in Table I. Inserting the shape parameters $\alpha_{\mathrm{HN}}$ and $\beta_{\mathrm{HN}}$ into the empirical relation involving $\alpha_{\mathrm{HN}}, \beta_{\mathrm{HN}}$ and $\beta_{K}$, $\beta_{K}=\left[\left(1-\alpha_{\mathrm{HN}}\right) \beta_{\mathrm{HN}}\right]^{1 / 1.23}[27]$, we obtain the stretching parameter $\beta_{K}$ in the relaxation function described by the stretched exponential function, $\phi(t)=\exp \left[-\left(t / \tau_{K}\right)^{\beta_{K}}\right]$. The values of $\beta_{K}$ obtained in this manner are $\beta_{K}=0.2$ and 0.3 for $d=18 \mathrm{~nm}$ and $247 \mathrm{~nm}$, respectively. Because the smaller value of $\beta_{K}$ means the broader distribution of the relaxation times, in this case, of the $\alpha$ process, this result qualitatively confirms the above experimental results obtained in the TES measurements.

\section{B. Glass transition temperature}

Figure 2 displays the $d$ dependence of $T_{\alpha}$ for a-PS films with three different molecular weights for the modulation frequency of temperature $f_{T}=8.3 \mathrm{mHz}$. Since the relaxation time corresponding to this frequency is of the order of $10^{2} \mathrm{sec}$, i.e., a macroscopic time scale, the temperature $T_{\alpha}$ can be regarded as the glass transition temperature $T_{\mathrm{g}}$. This definition of $T_{\mathrm{g}}$ has been supported by several experiments. For example, Hensel and Schick showed that the calorimetric

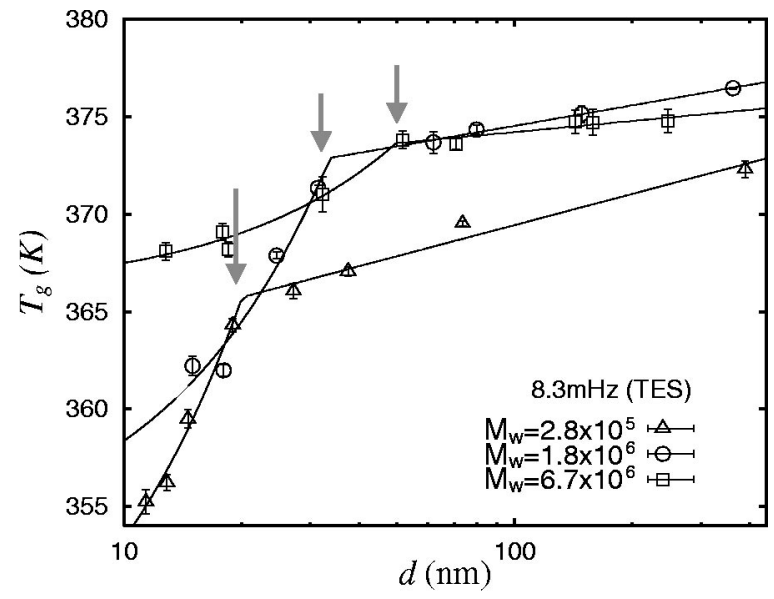

FIG. 2. Thickness dependence of the glass transition temperature $T_{\mathrm{g}}$. The value of $T_{\mathrm{g}}$ is measured as the temperature $T_{\alpha}$ at which the imaginary part of thermal expansion coefficients has a peak value for the modulation frequency $8.3 \mathrm{mHz}$ in TES measurements. The arrows show the crossover thickness $d_{c}$, below that $T_{\mathrm{g}}$ decreases abruptly with decreasing film thickness. $M_{\mathrm{w}}=2.8$ $\times 10^{5}(\triangle), 1.8 \times 10^{6}(\bigcirc)$, and $6.7 \times 10^{6}(\square)$.
TABLE II. Critical thickness $d_{c}$ and radius of gyration $R_{\mathrm{g}}$ of a-PS $\left(M_{\mathrm{w}}=2.8 \times 10^{5}, 1.8 \times 10^{6}\right.$, and $\left.6.7 \times 10^{6}\right)$.

\begin{tabular}{lccc}
\hline \hline$M_{\mathrm{w}}$ & $2.8 \times 10^{5}$ & $1.8 \times 10^{6}$ & $6.7 \times 10^{6}$ \\
\hline$d_{c}(\mathrm{~nm})$ & 20 & 34 & 50 \\
$R_{\mathrm{g}}(\mathrm{nm})$ & 16 & 41 & 79 \\
\hline
\end{tabular}

glass transition temperature determined by DSC (differential scanning calorimetry) during linear cooling with the rate of $-10 \mathrm{~K} / \mathrm{min}$ corresponds to the temperature at which a structural relaxation time is about $100 \mathrm{sec}$ by temperaturemodulated DSC [28]. Bauer et al. measured the glass transition temperatures using capacitive high-frequency detection in temperature ramping as well as in harmonic temperature cycling experiments. They found that $T_{\mathrm{g}}$ determined during cooling with the rate of $-10 \mathrm{~K} / \mathrm{min}$ corresponds to an average structural relaxation time of about $700 \mathrm{sec}$ [16].

According to the above definition we can see in Fig. 2 that the glass transition temperature thus obtained decreases slightly with decreasing film thickness down to a critical thickness $d_{c}$. Below $d_{c}, T_{\mathrm{g}}$ decreases much more rapidly with decreasing film thickness. The $d$ dependence of $T_{\mathrm{g}}$ below $d_{c}$ seems to be fitted by a linear function of $d$. (Note that the horizontal axis is on a logarithmic scale in Fig. 2.) Furthermore, the $d$ dependence of $T_{\mathrm{g}}$ clearly depends on the molecular weight of the polymer. As $M_{\mathrm{w}}$ increases, the critical thickness $d_{c}$ increases and the slope of $T_{\mathrm{g}}$ with $d$ below $d_{c}$ also changes with $M_{\mathrm{w}}$.

As listed in Table II, the values of $d_{c}$ change with $M_{\mathrm{w}}$ in accordance with the radius of gyration $R_{\mathrm{g}}$ [29]. This implies the existence of strong correlation between $d_{c}$ and $R_{\mathrm{g}}$. The $M_{\mathrm{w}}$ and $d$ dependence observed in the present measurements is similar to that of $T_{\mathrm{g}}$ observed in freely standing films of a-PS $[9,15]$ and also to that of $T_{\alpha}$ in supported films observed above $T_{\mathrm{g}}$ by using dielectric relaxation spectroscopy in the frequency range from $10^{2} \mathrm{~Hz}$ to $10^{4} \mathrm{~Hz}[7,8]$. From the experimental results it follows that the $M_{\mathrm{w}}$ and $d$ dependence can be ascribed to the confinement effects of polymer chains into thin film geometry. This effect is characteristic of the dynamics of the $\alpha$ process of thin polymer films and is absent in small molecules in confined geometry.

\section{Relaxation rate of the $\alpha$ process and Vogel temperature}

Figure 3 displays the dependence of the relaxation rate of the $\alpha$ process, $f_{\mathrm{m}}\left(\equiv 1 / 2 \pi \tau_{\alpha}\right.$, where $\tau_{\alpha}$ is the relaxation time), on temperature for thin a-PS films with various film thicknesses. We measured the temperature $T_{\alpha}$ at a given frequency $f_{\mathrm{m}}\left(=f_{E}\right.$ for DES or $f_{T}$ for TES) using the DES and TES methods and combined the two data to obtain the relaxation rate $f_{\mathrm{m}}$ in a wider frequency range from $10^{-3} \mathrm{~Hz}$ to $10^{4} \mathrm{~Hz}$. The upper and lower figures are the results for $M_{\mathrm{w}}$ $=1.8 \times 10^{6}$ and $6.7 \times 10^{6}$, respectively. We can see that at a given temperature above $390 \mathrm{~K}$, the relaxation rate $f_{\mathrm{m}}$ increases with decreasing film thickness. At a temperature near the glass transition (for examples, $370 \mathrm{~K}$ ), the thickness dependence of $f_{\mathrm{m}}$ is much more enhanced, i.e., there is a larger shift in $f_{\mathrm{m}}$ near $T_{\mathrm{g}}$ than at higher temperatures for the same 

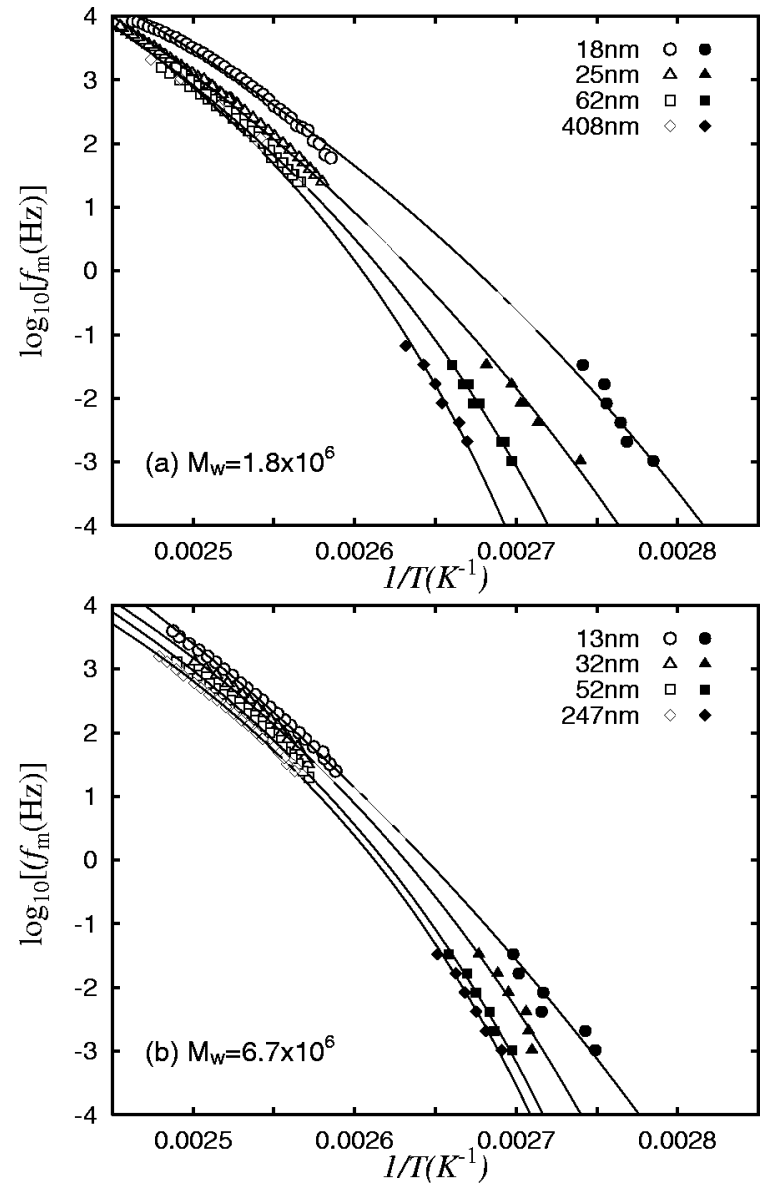

FIG. 3. Dispersion map for thin films of a-PS obtained from the peak positions of the loss component $\alpha_{\mathrm{n}}^{\prime \prime}$ or $\epsilon^{\prime \prime}$ has the peak value for various film thicknesses. The value of $f_{\mathrm{m}}$ satisfies the relation $2 \pi f_{\mathrm{m}} \tau_{\alpha}=1$, where $\tau_{\alpha}$ is the relaxation time of the $\alpha$ process. Full and open symbols stand for the values measured by TES and DES, respectively. The thicknesses are shown in the figure. The solid curve is calculated by using the VFT equation. (a) $M_{\mathrm{w}}=1.8 \times 10^{6}$, (b) $M_{\mathrm{w}}=6.7 \times 10^{6}$.

amount of change in thickness. From this result it follows that the thickness at which the finite size effect begins to appear increases as the temperature approaches the glass transition temperature. This fact is consistent with the physical picture of Adam and Gibbs that the size of the cooperatively rearranging region (CRR), $\xi_{\mathrm{CRR}}$ increases as the temperature approaches $T_{\mathrm{g}}$ from higher temperatures [30], where the CRR is a domain in which all molecules move cooperatively. If the system size becomes smaller and is comparable to $\xi_{\mathrm{CRR}}$, the dynamics should change significantly from those in the bulk system. It is expected that as $\xi_{\mathrm{CRR}}$ increases the thickness at which any change in the dynamics occurs becomes larger. The present experimental result of $f_{\mathrm{m}} v s 1 / T$ does qualitatively agree with the model with the growing $C R R$. This behavior has been observed in thin a-PS films with the two different molecular weights. The similar system size dependence of the relaxation rate of the $\alpha$ process has also been observed for the $\alpha$ process of small molecules confined in nanopores by using dielectric relaxation spectros-

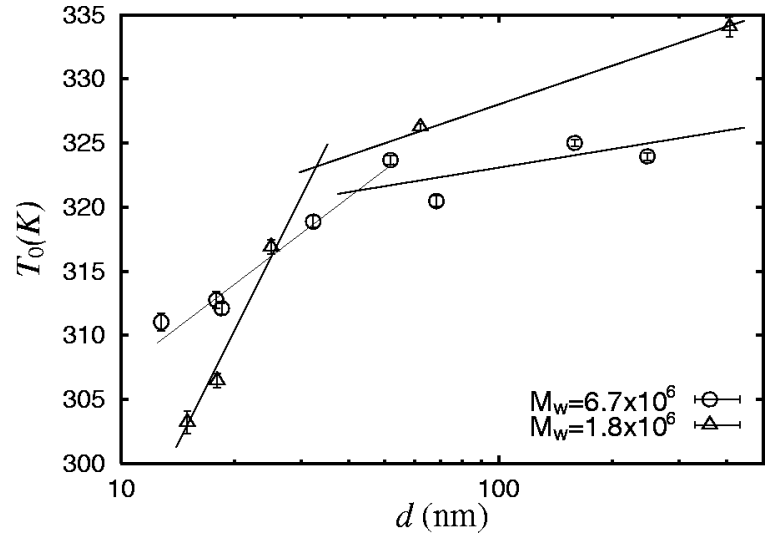

FIG. 4. Thickness dependence of the Vogel temperature $T_{0}$ for thin films of a-PS $\left(\bigcirc, M_{\mathrm{w}}=6.7 \times 10^{6} ; \triangle, M_{\mathrm{w}}=1.8 \times 10^{6}\right)$. The values of $T_{0}$ are obtained by fitting $f_{\mathrm{m}}$ vs $1 / T$ to Eq. (6) on condition that the parameter $\tau_{0}$ is independent of thickness. The solid lines are guides for eyes.

copy [31]. Such a system size dependence may be characteristic of the dynamics of the confined geometry.

In Fig. 3 it can be seen that there is the molecular weight dependence of the relaxation dynamics of the $\alpha$ process in thinner films. In order to extract the molecular weight and thickness dependence of $f_{\mathrm{m}}$, the observed values of $f_{\mathrm{m}}$ are fitted to the VFT law and the parameters $U$ and $T_{0}$ are obtained as functions of $d$ for two different molecular weights. We tried fitting procedures in two different ways: (1) all the three fitting parameters $\tau_{0}, U$, and $T_{0}$ are adjusted to reproduce the observed values, (2) two parameters $U$ and $T_{0}$ are adjusted on condition that the parameter $\tau_{0}$ is fixed to be a value obtained for the bulk films. The assumption in the case (2) corresponds to the one that $\tau_{0}$ is independent of thickness, i.e., the relaxation times at high temperatures are the same regardless of thickness.

Figure 4 displays the thickness dependence of $T_{0}$ for thin films of a-PS with $M_{\mathrm{w}}=1.8 \times 10^{6}$ and $6.7 \times 10^{6}$. The values of $T_{0}$ in Fig. 4 are obtained by the fitting procedure (2). The value of $\tau_{0}$ is fixed to be $2.42 \times 10^{-13} \mathrm{sec}$ and 2.87 $\times 10^{-14} \mathrm{sec}$ for $M_{\mathrm{w}}=1.8 \times 10^{6}$ and $6.7 \times 10^{6}$, respectively. The values are obtained by fitting the thickness dependence of $f_{\mathrm{m}}$ for the bulk sample to Eq. (6). In Fig. 4 we can see that the Vogel temperature decreases with decreasing thickness in the similar way to the glass transition temperature (Fig. 2) except that there is the difference $(\sim 50 \mathrm{~K})$ in absolute values between $T_{0}$ and $T_{\mathrm{g}}$ at a fixed thickness. Furthermore, we can see in Fig. 4 that $T_{0}$ also depends on the molecular weight of the polymers.

It should be noted that the $M_{\mathrm{w}}$ dependence of $T_{0}$ could not be obtained beyond the error bars by the fitting procedure (1), although it is confirmed that the thickness dependence of $T_{0}$ could clearly be observed, i.e., $T_{0}$ decreases with decreasing thickness whichever fitting procedure is chosen.

\section{Thermal expansion coefficient of the free volume}

Figure 5 displays $1 / U$ as a function of $1 / d$, where the values of $U$ are obtained by the fitting procedure (2). In Fig. 


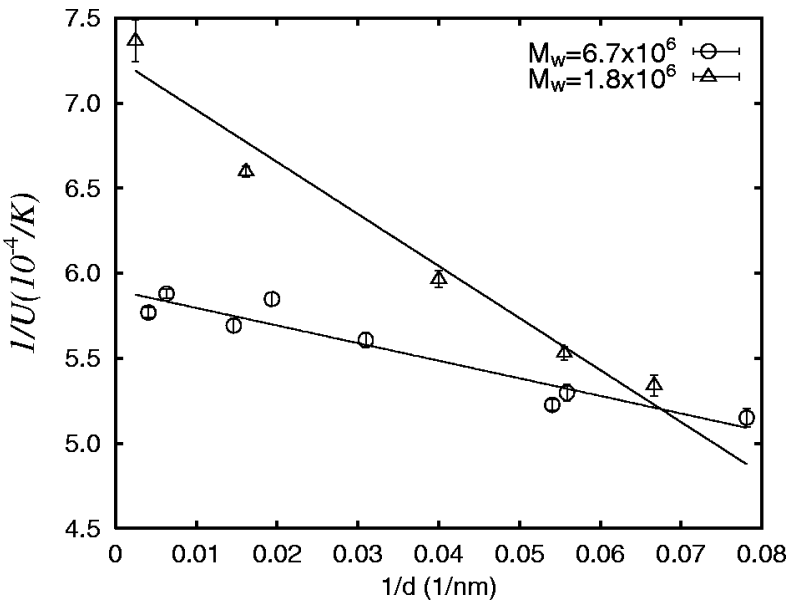

FIG. 5. The dependence of $1 / U$ on $1 / d$ for thin films of a-PS ( $\bigcirc, M_{\mathrm{w}}=6.7 \times 10^{6} ; \triangle, M_{\mathrm{w}}=1.8 \times 10^{6}$ ). The thermal expansion coefficient of the free volume $\alpha_{f}$ is proportional to $1 / U$ within the free volume theory. The straight lines are reproduced by Eq. (11).

5 , it is found that $1 / U$ decreases as $1 / d$ increases, i.e., the apparent activation energy $U$ increases with decreasing film thickness. Here we will analyze the present result within the free volume theory.

In the free volume theory of Cohen-Turnbull [32], the relaxation time of the $\alpha$ process can be described by the following relation:

$$
\tau_{\alpha}=\tau_{0} \exp \left(\frac{b}{\tilde{f}}\right)
$$

where $\widetilde{f}$ is the fraction of the free volume and $b$ is a constant. If we assume that the fraction $\widetilde{f}$ increases linearly with temperature as

$$
\tilde{f}=f_{\mathrm{g}}+\alpha_{f}\left(T-T_{\mathrm{g}}\right)
$$

we obtain

$$
\tau_{\alpha}=\tau_{0} \exp \left(\frac{b}{\alpha_{f}\left(T-T_{\mathrm{g}}\right)+f_{\mathrm{g}}}\right),
$$

where $\alpha_{f}$ is the thermal expansion coefficient of free volume and $f_{\mathrm{g}}$ is the free volume fraction at $T_{\mathrm{g}}$. Comparing Eq. (6) with Eq. (9), we obtain the Vogel temperature $T_{0}$ and the thermal expansion coefficient of the free volume $\alpha_{f}$ in the following way:

$$
T_{0}=T_{\mathrm{g}}-\frac{f_{\mathrm{g}}}{\alpha_{f}} \quad \text { and } \quad \alpha_{f}=\frac{b}{U}
$$

Because $\alpha_{f}$ is proportional to $1 / U$, we can see in Fig. 5 that the thermal expansion coefficient of the free volume $\alpha_{f}$ decreases with decreasing film thickness. As shown in the straight line in Fig. 5, the dependence of $\alpha_{f}$ on $d$ is given as follows:

$$
\alpha_{f}=\alpha_{f}^{\infty}\left(1-\frac{a}{d}\right)
$$

where $\alpha_{f}^{\infty}$ is the thermal expansion coefficient of the free volume in the bulk system and $a$ is a characteristic length. It is determined that the values of $a$ are $4.2 \pm 0.3 \mathrm{~nm}$ and 1.8 $\pm 0.2 \mathrm{~nm}$ for $M_{\mathrm{w}}=1.8 \times 10^{6}$ and $6.7 \times 10^{6}$, respectively. This result suggests that within the free volume theory the mobility related to the thermal expansion of the free volume is reduced in thinner films compared with that in the bulk system.

The thermal expansion coefficient of the free volume has directly been measured for thin films of a-PS by using PALS [6]. The result shows that the thermal expansion coefficient decreases with decreasing thickness in the same manner described by Eq. (11). The obtained value of $a$ is 5.0 $\pm 0.5 \mathrm{~nm}$ in the case of PALS. Furthermore, our previous measurements on temperature change in the electric capacitance show that the thermal expansion coefficient of thin films of a-PS above $T_{\mathrm{g}}$ also obeys Eq. (11) and $a=2.5$ $\pm 0.3 \mathrm{~nm}[8]$. Thus, the PALS and the capacitance measurements on thin films of a-PS support the validity of Eq. (11), which is extracted from the observed values of $f_{\mathrm{m}}$ within the free volume theory.

Taking account of the $d$ dependence of $\alpha_{f}$ and $T_{0}$, we obtain the relations for two different thicknesses $d_{1}$ and $d_{2}$,

$$
\begin{aligned}
& \widetilde{f}\left(d_{1} ; T\right)=\alpha_{f}\left(d_{1}\right)\left[T-T_{0}\left(d_{1}\right)\right], \\
& \widetilde{f}\left(d_{2} ; T\right)=\alpha_{f}\left(d_{2}\right)\left[T-T_{0}\left(d_{2}\right)\right] .
\end{aligned}
$$

The present measurement shows that the decrease in $\alpha_{f}$ $(\sim 1 / U)$, which is associated with the slower relaxation, competes with the decrease in $T_{0}$ in thin films, which can be regarded as the speedup of the $\alpha$ process. Hence, we find that at a finite (crossover) temperature $T^{*}$ the two straight lines corresponding to $d_{1}$ and $d_{2}$ meet with each other in the $T-\tilde{f}$ plane. This result leads to the conjecture that the relaxation dynamics of the $\alpha$ process in thinner films become slower than in the bulk system above $T^{*}$, although the relaxation dynamics are faster in thinner films in the temperature range investigated in the present measurements. If we perform the similar measurements at still higher temperatures, we will be able to confirm the existence of the temperature $T^{*}$ at which the $\alpha$-relaxation time in thin films becomes equal to that in the bulk system. Using the present data the value of $T^{*}$ can be estimated: for example, $T^{*}=431.6 \mathrm{~K}$ for $d_{1}=12.8 \mathrm{~nm}$ and $d_{2}=247.1 \mathrm{~nm}$ in thin films of PS with $M_{\mathrm{w}}=6.7 \times 10^{6}$.

Tseng et al. observed fluorescence recovery after photo bleaching in thin polystyrene films supported on fused quartz and reported that the diffusion constant $D$ of dye in thin PS films falls below the bulk values above $423 \mathrm{~K}$, although $D$ increases with decreasing film thickness at a given temperature below $423 \mathrm{~K}$ [33].

In the previous paper [8], we found that the existence of a dead layer, where molecular mobility is highly suppressed 

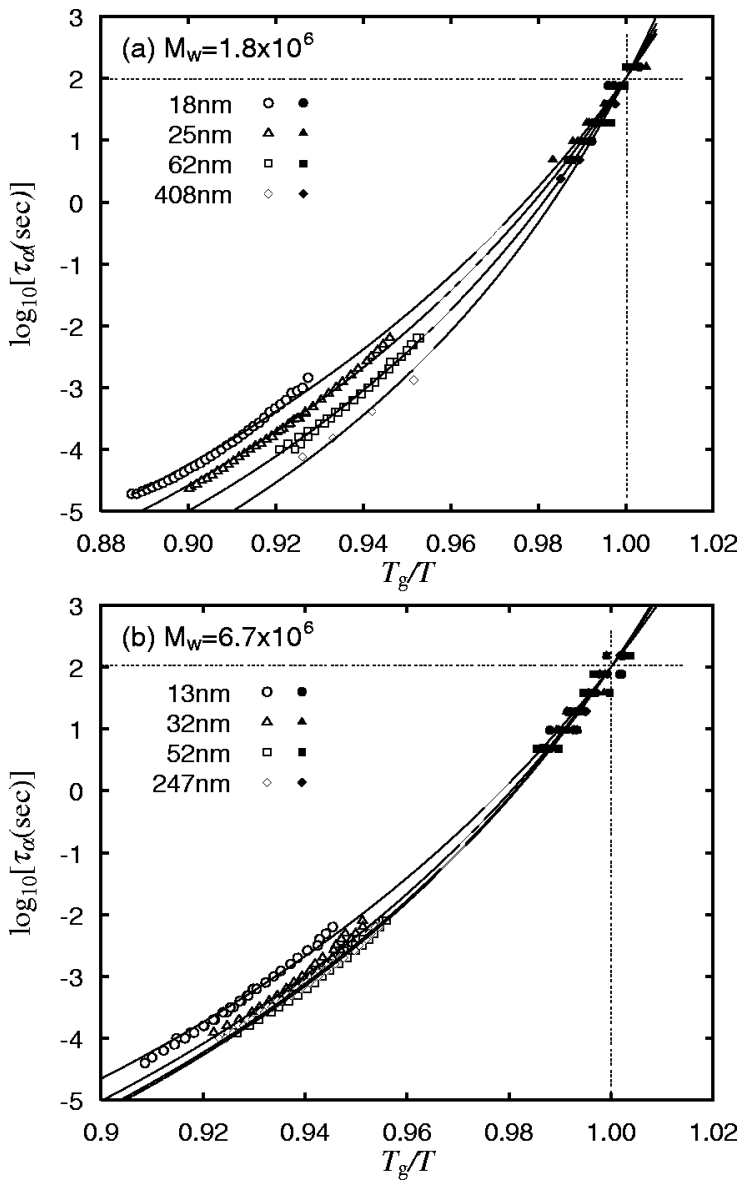

FIG. 6. The dependence of $\log _{10} \tau_{\alpha}$ on the inverse of the reduced temperature $T_{\mathrm{g}} / T$ for thin films of a-PS, (a) $M_{\mathrm{w}}=1.8 \times 10^{6}$, (b) $M_{\mathrm{w}}=6.7 \times 10^{6}$. The values of $T_{\mathrm{g}}$ are obtained by the relation $\tau_{\alpha}\left(T_{\mathrm{g}}\right)=10^{2} \mathrm{sec}$. The symbols are the same in Fig. 3 .

compared with that in a bulklike layer, is essential to reproduce the observed thickness dependence of the thermal expansion coefficient above $T_{\mathrm{g}}$. Equation (11) suggests the existence of a similar dead layer, which does not contribute to the thermal expansion of the free volume. The observed reduction in chain mobility in thin supported polymer films $[13,14]$ may be due to the existence of the dead layer.

\section{E. Fragility}

As shown in the previous section, the apparent activation energy $U$ of the $\alpha$ process increases with decreasing film thickness. From this result, it is expected that the fragility $m$ changes with film thickness. Here, the fragility is a measure of non-Arrhenius character and is defined as follows [34]:

$$
m=\left[\frac{d \log \tau_{\alpha}(T)}{d\left(T_{\mathrm{g}} / T\right)}\right]_{T=T_{\mathrm{g}}} .
$$

The observed relaxation rate of the $\alpha$ process is converted into the relaxation time using the relation $2 \pi f_{\mathrm{m}} \tau_{\alpha}=1$. Furthermore, the glass transition temperature $T_{\mathrm{g}}$ is redefined as the temperature at which the relation $\tau_{\alpha}\left(T_{\mathrm{g}}\right)=10^{2} \mathrm{sec}$ is satisfied. Figure 6 shows the Angell plot of the relaxation time

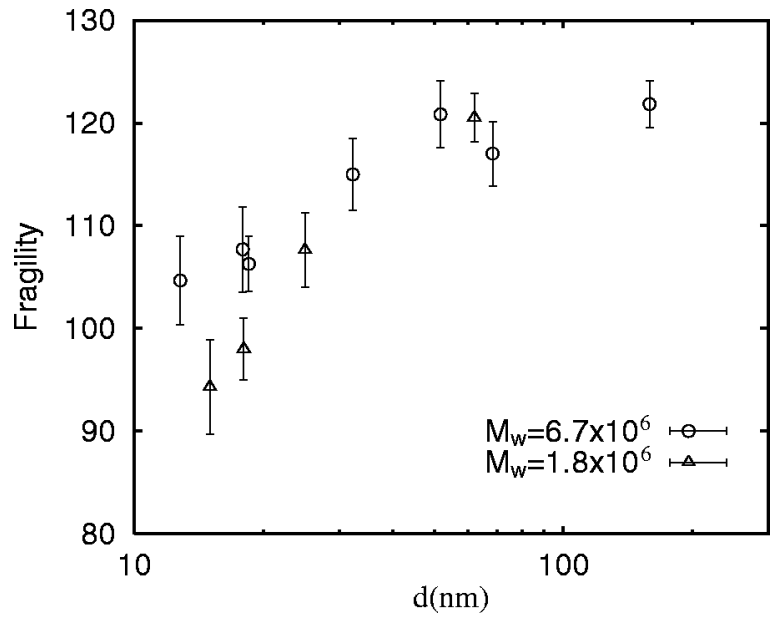

FIG. 7. Thickness dependence of fragility index $m$ for thin films of a-PS, $M_{\mathrm{w}}=1.8 \times 10^{6}(\triangle), M_{\mathrm{w}}=6.7 \times 10^{6}(\bigcirc)$.

$\tau_{\alpha}$ of the $\alpha$ process in thin films of a-PS, where the values of $\log _{10} \tau_{\alpha}$ are plotted as a function of $T_{\mathrm{g}} / T$. In this figure, the fragility index is obtained as the slope of the tangential line at $T_{\mathrm{g}}$.

Figure 7 shows the thickness dependence of the fragility thus obtained for two different molecular weights. In this figure we can see that the fragility decreases with decreasing film thickness, i.e., thin films of a-PS become less fragile (more strong) as the thickness decreases. This result means that the temperature dependence of $\tau_{\alpha}$, which can be described by the VFT law, approaches the simple Arrhenius law as the thickness decreases. If it is expected that the origin of the non-Arrhenius behavior according to the VFT law is related to the cooperativity in the dynamics of the $\alpha$ process, the observed thickness dependence of fragility index leads to the conjecture that the dynamics of the $\alpha$ process in thin films change from the cooperative dynamics as observed in normal liquid states towards the single molecular dynamics.

In small molecules confined in nanopores, it is observed by dielectric relaxation spectroscopy that the temperature dependence of the relaxation time of the $\alpha$ process is described by the Arrhenius law for ethylene glycol confined in zeolite with pore size less than $0.5 \mathrm{~nm}[35,36]$. From this result, Kremer et al. estimated the minimum number of molecules required for the emergence of non-Arrhenius character of liquids. The present result shows that there is the tendency towards the single molecular dynamics in polymers in thin films as well as in simple molecules confined in nanopores.

\section{CONCLUDING REMARKS}

In this paper, we have investigated the dynamics of the $\alpha$ process in thin films of a-PS in the frequency range from $10^{-3} \mathrm{~Hz}$ to $10^{4} \mathrm{~Hz}$ by using dielectric relaxation spectroscopy and thermal expansion spectroscopy. The results obtained in this study can be summarized as follows.

(1) We have successfully observed the molecular weight dependence of the glass transition temperature for thin a-polystyrene films supported on glass substrate and found 
the existence of a crossover thickness at which the thickness dependence of $T_{\mathrm{g}}$ changes abruptly.

(2) The crossover thickness $d_{c}$ strongly depends on the molecular weight of the polymer; the values of $d_{c}$ change in the similar way to the radius of gyration of the polymer chain $\left(d_{c} \sim R_{\mathrm{g}}\right)$.

(3) The relaxation time of the $\alpha$ process $\tau_{\alpha}$ decreases with decreasing thickness. The thickness dependence of $\tau_{\alpha}$ is much more prominent near the glass transition.

(4) The Vogel temperature $T_{0}$ decreases with decreasing thickness.

(5) The thermal expansion coefficient of the free volume $\alpha_{f}$ decreases with decreasing thickness within the free volume theory.

(6) The fragility index $m$ decreases with decreasing thickness.

The result (2) implies that the chain confinement effect on the dynamics of the $\alpha$ process may be involved in the glass transition behavior in thin polymer films. The molecular weight dependence of the crossover thickness in thin polymer films supported on glass substrate is qualitatively the same as that observed in freely standing films of polymers [10].

In order to explain the existence of the crossover thickness associated strongly with $R_{\mathrm{g}}$, de Gennes proposed a model in which a new mode of chain motions called sliding motion is introduced in addition to the segmental motion
$[37,38]$. This new motion is a collective motion along the chain, which requires a smaller free volume except for the end groups. De Gennes shows that in the bulk state the sliding motion is suppressed because of the end group hindrance below $T_{\mathrm{g}}$, while in thinner films $\left(d<R_{\mathrm{g}}\right)$ this motion is activated through soft surface layers even below $T_{\mathrm{g}}$. Thus, some properties of $T_{\mathrm{g}}$ in the freely standing films of polystyrene can qualitatively be reproduced by de Gennes' model.

Although in the case of thin supported polymer films the situations are more complicated because of the interaction between the substrate and the polymers, the similar molecular weight dependence of $d_{c}$ has been observed as shown in the previous section. Hence, it is expected that de Gennes' model may partly be applicable also in the present case. At the same time, the present form of the model cannot explain the observed thickness and molecular weight dependence of $T_{0}$. We hope that the model will be developed in full agreement with many aspects of the glass transition in thin polymer films.

\section{ACKNOWLEDGMENTS}

The work was partly supported by a Grant-in-Aid (Nos. 11640395 and 12874048) from the Ministry of Education, Science, Sports, and Culture of Japan, and a Grant-in-Aid for Scientific Research on Priority Areas, Mechanism of Polymer Crystallization (No. 12127203).
[1] Proceedings of the International Workshop on Dynamics in Confinement, edited by B. Frick, R. Zorn, and H. Büttner [J. Phys. IV 10, 7 (2000).

[2] J. A. Forrest and R. A. L. Jones, in Polymer Surfaces, Interfaces, and Thin Films, edited by A. Karim and S. Kumar (World Scientific, Singapore, 2000)

[3] J. L. Keddie, R. A. L. Jones, and R. A. Cory, Europhys. Lett. 27, 59 (1994).

[4] J. L. Keddie and R. A. L. Jones, Faraday Discuss. 98, 219 (1994).

[5] W. E. Wallace, J. H. van Zanten, and W. L. Wu, Phys. Rev. E 52, R3329 (1995).

[6] G. B. DeMaggio, W. E. Frieze, D. W. Gidley, Ming Zhu, H. A. Hristov, and A. F. Yee, Phys. Rev. Lett. 78, 1524 (1997).

[7] K. Fukao and Y. Miyamoto, Europhys. Lett. 46, 649 (1999).

[8] K. Fukao and Y. Miyamoto, Phys. Rev. E 61, 1743 (2000).

[9] J. A. Forrest, K. Dalnoki-Veress, J. R. Stevens, and J. R. Dutcher, Phys. Rev. Lett. 77, 2002 (1996).

[10] J. A. Forrest, K. Dalnoki-Veress, and J. R. Dutcher, Phys. Rev. E 56, 5705 (1997).

[11] D. B. Hall, J. C. Hooker, and J. M. Torkelson, Macromolecules 30, 667 (1997).

[12] J. A. Forrest, C. Svanberg, K. Revesz, M. Rodahl, L. M. Torell, and B. Kasemo, Phys. Rev. E 58, R1226 (1998).

[13] B. Frank, A. P. Gart, T. P. Russell, H. R. Brown, and C. Hawker, Macromolecules 29, 6531 (1996).

[14] X. Zheng, B. B. Sauer, J. G. Van Alsten, S. A. Schwarz, M. H. Rafailovich, J. Sokolov, and M. Rubinstein, Phys. Rev. Lett. 74, 407 (1995).
[15] K. Dalnoki-Veress, J. A. Forrest, C. Murray, C. Gigault, and J. R. Dutcher, Phys. Rev. E 63, 031801 (2001).

[16] C. Bauer, R. Böhmer, S. Moreno-Flores, R. Richert, H. Sillescu, and D. Neher, Phys. Rev. E 61, 1755 (2000).

[17] C. Bauer, R. Richert, R. Böhmer, and T. Christensen, J. NonCryst. Solids 262, 276 (2000).

[18] K. Fukao and Y. Miyamoto, J. Phys. IV 10, 243 (2000).

[19] P. K. Dixon, L. Wu, S. R. Nagel, B. D. Williams, and J. Carini, Phys. Rev. Lett. 65, 1108 (1990).

[20] A. Schönhals, F. Kremer, A. Hofmann, E. W. Fischer, and E. Schlosser, Phys. Rev. Lett. 70, 3459 (1993).

[21] P. Lunkenheimer, A. Pimenov, M. Dressel, Yu. G. Goncharov, R. Böhmer, and A. Loidl, Phys. Rev. Lett. 77, 318 (1996).

[22] O. Yano and Y. Wada, J. Polym. Sci., Part A-2 9, 669 (1971).

[23] G. Blum, F. Kremer, T. Jaworek, and G. Wegner, Adv. Mater. 7, 1017 (1995).

[24] S. Kawana and R. A. L. Jones, Phys. Rev. E 63, 021501 (2001).

[25] S. Havriliak and S. Negami, Polymer 8, 161 (1967).

[26] H. Vogel, Phys. Z. 22, 645 (1921); G. S. Fulcher, J. Am. Ceram. Soc. 8, 339,789 (1925).

[27] F. Alvarez, A. Alegria, and J. Colmenero, Phys. Rev. B 44, 7306 (1991).

[28] A. Hensel and C. Schick, J. Non-Cryst. Solids 235-237, 510 (1998).

[29] Polymer Handbook, 4th ed., edited by J. Brandrup, E. H. Immergut, and E. A. Grulke (Wiley, New York, 1999), Sec. $\mathrm{VII} / 1$. 
[30] G. Adam and J. H. Gibbs, J. Chem. Phys. 43, 139 (1965).

[31] M. Arndt, R. Stannarius, H. Groothues, E. Hempel, and F. Kremer, Phys. Rev. Lett. 79, 2077 (1997).

[32] M. H. Cohen and D. Turnbull, J. Chem. Phys. 31, 1164 (1959).

[33] K. C. Tseng, N. J. Turro, and C. J. Durning, Phys. Rev. E 61, 1800 (2000).

[34] R. Böhmer and C. A. Angell, Phys. Rev. B 45, 10091 (1992).
[35] A. Huwe, F. Kremer, P. Behrens, and W. Schwieger, Phys. Rev. Lett. 82, 2338 (1999).

[36] F. Kremer, A. Huwe, M. Arndt, P. Behrens, and W. Schwieger, J. Phys.: Condens. Matter 11, A175 (1999).

[37] P. G. de Gennes, Eur. Phys. J. E 2, 201 (2000).

[38] P. G. de Gennes, C. R. Acad. Sci. IV-Phys. 1, 1179 (2000). 